\title{
Temperature wave-like oscillations on ultra-short and ultra-fast time scales -INVITED
}

\author{
Gandolfi Marco ${ }^{1,2}$, Benetti Giulio ${ }^{3}$, Glorieux Christ ${ }^{1}$, Giannetti Claudio ${ }^{2}$, Banfi Francesco $^{4, *}$ \\ ${ }^{1}$ Laboratory of Soft Matter and Biophysics, Department of Physics and Astronomy, KU Leuven, Celestijnenlaan 200D, B-3001 Leuven, \\ Belgium \\ ${ }^{2}$ Interdisciplinary Laboratories for Advanced Materials Physics (I-LAMP) and Dipartimento di Matematica e Fisica, Università Cattolica \\ del Sacro Cuore, Via Musei 41, 25121 Brescia, Italy \\ ${ }^{3}$ Department of Pathology and Diagnostics - Medical Physics Unit, Azienda Ospedaliera Universitaria Integrata - Verona, P.le Stefani, \\ 1,37126 Verona, Italy \\ ${ }^{4}$ Université de Lyon, Institut Lumière Matière (iLM), Université Lyon 1 and CNRS, 10 rue Ada Byron, 69622 Villeurbanne cedex, \\ France.
}

\begin{abstract}
Recent findings in the frame of temperature wave-like oscillations on the ultra-short, ultra-fast time scales in solid states devices are here reviewed. The possibility for wave-like temperature oscillations are investigated at the light of the pass-band characteristic in w-k space for the temperature scalar field. The bandpass filter characteristics are accessed in terms of the heat carriers scattering times. The concepts here reviewed are of interest for perspective design of novel thermal nano-devices.
\end{abstract}

\section{Introduction}

Coherent control of wave-like phenomena is driving a technological revolution in fields ranging from electronics, photonics, to phononics 1.

Although temperature has been historically taken as the paradigmatic example of an incoherent field, undergoing diffusive as opposed to wave-like propagation, on short space and time scales the Fourier law fails and the possibility for temperature wave propagation sets in (1) The ultimate goal is to devise materials exploiting the wave nature of the temperature scalar field arising in the non-Fourier heat transport regime. We here review recent progress made in this context [4-6]

\section{Discussion}

The Dual Phase Lag (DPL) model (2) has been introduced to correct for the instantaneous onset of the heat flux, q, upon establishment of a thermal gradient, $\nabla \mathrm{T}$, entailed in Fourier law. The DPL model generalizes earlier attempts - made by Cattaneo (1958) and Vernotte (1962) - to correct this pitfall by introducing causality between the cause $\nabla T(q)$, and the effect, $q(\nabla T)$ :

$$
\mathrm{q}\left(\mathrm{x}, \mathrm{t}+\tau_{\mathrm{q}}\right)=-\mathrm{k}_{\mathrm{T}} \nabla \mathrm{T}\left(\mathrm{x}, \mathrm{t}+\tau_{\mathrm{T}}\right)
$$

In cases where $\tau_{\mathrm{q}}<\tau_{\mathrm{T}}$ the heat flux is the cause, whereas if $\tau_{\mathrm{q}}>\tau_{\mathrm{T}}$ the temperature gradient is the cause. Coupling the DPL with thermal energy conservation, the wellknown heat diffusion equation (parabolic) is modified into a hyperbolic one:

$$
\left(\frac{\tau_{\mathrm{q}}}{\alpha}\right) \frac{\partial^{2} \mathrm{~T}}{\partial \mathrm{t}^{2}}-\frac{\partial^{2} \mathrm{~T}}{\partial \mathrm{x}^{2}}+\left(\frac{1}{\alpha}\right) \frac{\partial \mathrm{T}}{\partial \mathrm{t}}-\tau_{\mathrm{T}} \frac{\partial^{3} \mathrm{~T}}{\partial \mathrm{x}^{2} \partial \mathrm{t}}=0
$$

, where $\alpha=\mathrm{k}_{\mathrm{T}} / \mathrm{C}$ is the thermal diffusivity. The hyperbolic equation encompasses wave-terms (first twoterms) with damping (last two terms). The parabolic case is retrieved on "long" time scales when the second and third terms dominate.

Recently a user-friendly, intuitive approach [4] has been proposed to investigate under which conditions the temperature propagation safeguards coherent (wave-like) effects. This is achieved calculating the complex dispersion relation, $f(k, \omega)=0$, for the hyperbolic equation linking the wave vectors $\mathrm{k}$ to the angular frequency $\omega$. Specifically, the case $\omega \in \mathbb{C}$ and $k \in \mathbb{R}$ describes the physical situation of a temperature pulse propagating in the material, where the real part $\omega_{1}=$ $\mathcal{R e}(\omega)$ is an oscillation angular frequency, whereas the imaginary part $\omega_{2}=\mathfrak{I m}(\omega)$ has the physical meaning of damping. Wave-like oscillations are sustained provided the quality factor $Q=\omega_{1} / \omega_{2}>1$. Materials sustaining temperature waves on short timescales are addressed, in the current work as Temperonic materials.

In general a material can sustain the following dynamics: (i) overdamped, $\mathrm{Q}<1$, (no transient temperature oscillations sustained) when $\mathrm{q}$ is the cause $\left(\tau_{\mathrm{T}} / \tau_{\mathrm{q}}>1\right)$ (ii) either over, critically or underdamped when $\nabla \mathrm{T}$ is the cause $\left(\tau_{\mathrm{T}} / \tau_{\mathrm{q}}<1\right)$, the best case scenaro to observe transient temperature wave- behaviour being $\tau_{\mathrm{T}} / \tau_{\mathrm{q}}=0$ (Cattaneo-Vernotte limit).

The complex-valued $\omega-\mathrm{k}$ dispersion relation is investigated for the cases of a localised temperature pulse in space, linking the temperature wave angular frequencies $\omega$ and wave vectors $\mathrm{k}$. The modal Q-factor allows discerning which temperature oscillations modes are practically accessible and allows inspecting the material as a frequency filter for the propagation of temperature oscillations. The optimal region in parameters space is discussed in a variety of systems, ranging from graphite to quantum materials [4-6]. 


\section{Conclusions}

This presentation applies an easy-to-adopt analytical means to inspect the optimal conditions to observe temperature wave oscillations. The idea parallels the approach successfully employed in solid state physics and optics to investigate electronic wavefunction and electromagnetic wave propaga- tion in solid state devices. The calculations are applied in a variety of systems of technological interest. The present discussion is of relevance in view of engineering nanoscale thermal devices operating on ultra-fast and ultra-short time scales

\section{References}

1. M. Wegener, Science 342, 939 (2013)

2. M. Maldovan, Nature 503, 209 (2013)

3. D.Y. Tzou, Macro-to Microscale Heat Transfer: The Lagging Behavior (John Wiley \& Sons, 2014)

4. M. Gandolfi, G. Benetti, C. Glorieux, C. Giannetti, and F. Banfi, Int. J. Heat Mass Transf. 143, 118553 (2019)

5. M Gandolfi, G L Celardo, F Borgonovi, G Ferrini, A Avella, F Banfi and C Giannetti, Phys. Scr. 92, 034004 (2017)

6. S. Huberman, R.A. Duncan, K. Chen, B. Song, V. Chiloyan, Z. Ding, A.A. Maznev, G. Chen, K.A. Nelson, Science 364, (2019) 